\section{INTELLIGENT APP SOOTHES PATIENTS WITH MUSIC} prescribed soothing music chosen by artificial intelligence to ease their anxiety and pain, thanks to an encouraging trial of a health tech app on dementia patients.

British health tech start-up MediMusic has created an app and a streaming device called the MediBeat that dispenses personalised playlists to reduce anxiety and pain in patients using a 'digital drip' to administer the most calming music.

psychological science, it then compiles in seconds the perfect 20-minute playlist of soothing music to calm them.

Playlist running order is designed to reduce heart rate and stress hormones like cortisol and promotes relaxation through hormones like dopamine and oxytocin. The music is played through earphones and the MediBeat streaming device and a heart rate monitor worn on the wrist. The brain responds to music more than
Patients around the world will soon be any other stimulus. MediMusic's proprietary algorithms extract the relevant features from the digital DNA of a piece of music, resulting in a fingerprint for healthcare use.

Using artificial intelligence, machine learning and the data about the patient, MediMusic then automatically creates playlists from music streaming services within a couple of clicks and plays the music
It could revolutionise the treatment of dementia, pre/post operation, chronic pain, dentistry, and Alzheimer's Disease through to improving motor response as part of a physical rehabilitation programme.

Initial clinical NHS trials at Lancashire Teaching Hospitals NHS Foundation Trust found the use of MediMusic saw an up to $22 \%$ reduction in heart rate in patients with dementia during the COVID-19 pandemic. It can be used in hospitals, care homes and dentist surgeries and could slash medication bills by up to a quarter.

Music therapy has already been proven in several previous studies to reduce anxiety by $44 \%$ and pain by $28 \%$. This has resulted in a reduction in the need for relevant medication by $24 \%{ }^{1}$

Now the revolutionary treatment is undergoing an NHS trial also at Lancashire Teaching Hospitals NHS Foundation Trust, on 40 NHS doctors and nurses and other staff who have worked in critical care during the pandemic, to help them ease anxiety and stress.

The MediMusic app works with the patient's age, gender, nationality, and ethnicity and based on sociological and through a streaming device called the MediBeat and a pair of headphones. Each chosen track is 'heartrate optimised' to reduce anxiety, stress or pain, improve quality of life and streamline healthcare workflow.

A heart rate monitor worn on the wrist allows MediMusic to monitor the physiological effect of a piece of music upon a listener and if the listener's heart rate does not respond as expected, MediMusic's 'Digital Drip' uses artificial iIntelligence and machine learning to swap out forthcoming playlist tracks to invoke slower relaxation if required.

In addition to the track swap out process, MediMusic provides evidence based KPIs showing the service benefit and medication cost savings.

To find out more about MediMusic go to www.medimusic.co.

\section{Reference}

1. Guétin S, Giniès P, Siou D K A et al. The effects of music intervention in the management of chronic pain: a singleblind, randomized, controlled trial. Clin J Pain 2012; 28: 329-337.
SMART TOOTHBRUSH REMINDS PATIENTS

Worn toothbrushes remove less plaque. With Oral-B power toothbrushes, it is a combination of the head and handle configuration, which work in perfect synergy, to give the user the ultimate clean. The small round head mimics the action used in dental prophylaxis as it gently contours each tooth.

Convincing patients to change their toothbrush head regularly is not an easy task and can be a sensitive subject. Oral-B has launched a new brush head, called CleanMaximiser, which indicates when users should buy a replacement. Following dental professional recommendation, the bristles will change colour from green to yellow after three months of use, indicating it is time for a new brush head. The CleanMaximiser refills are compatible with all Oral-B models except the Pulsonic and iO.

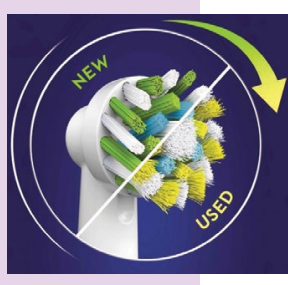

Created with wellbeing in mind, Heka's UNIC range of dental treatment centres combines aesthetics with functionality, creating the perfect environment for a pleasant visit to the dentist. To offer a quick snapshot of what UNIC can offer, the instrument table's handle is fully integrated with UNIC's display and touch pads. It is easy to find by both eye and hand - even when your attention is elsewhere.

With a light touch on the cuspidor touch pad, the suction stops, the operating light goes off and the patient's chair adjusts to the rinsing position.

Meanwhile, the glass fills with water. By touching the cuspidor touch pad again, the patient's chair returns to its previous working position.

Intrigued? Who wouldn't be?

If you would like to know more, simply contact Quintess Denta - visit https://bit.ly/3apfsAF, email info@ quintessdenta.com or call 0286862 8966 (UK) or 04868628966 (ROI). 